\title{
Effectiveness of airport screening at detecting travellers infected with novel coronavirus (2019-nCoV)
}

Billy J Quilty' ${ }^{1}$, Sam Clifford ${ }^{1}$, CMMID nCoV working group ${ }^{2}$, Stefan Flasche ${ }^{1,3}$, Rosalind M Eggo $0^{1,3}$

1. Centre for the Mathematical Modelling of Infectious Diseases, Department of Infectious Disease Epidemiology, London School of Hygiene and Tropical Medicine, London, United Kingdom

2. The members of the Centre for the Mathematical Modelling of Infectious Diseases (CMMID) nCoV working group are listed at the end of the article

3. These authors contributed equally to this work

Correspondence: Billy J Quilty (Billy.Quilty@lshtm.ac.uk)

Citation style for this article:

Quilty Billy J, Clifford Sam, CMMID nCoV working group, Flasche Stefan, Eggo Rosalind M . Effectiveness of airport screening at detecting travellers infected with novel coronavirus (2019-nCoV). Euro Surveill. 2020;25(5):pii=2000080. https://doi.org/10.2807/1560-7917.ES.2020.25·5.2000080

We evaluated effectiveness of thermal passenger screening for 2019-nCoV infection at airport exit and entry to inform public health decision-making. In our baseline scenario, we estimated that $46 \%$ (95\% confidence interval: 36 to 58 ) of infected travellers would not be detected, depending on incubation period, sensitivity of exit and entry screening, and proportion of asymptomatic cases. Airport screening is unlikely to detect a sufficient proportion of 2019-nCoV infected travellers to avoid entry of infected travellers.

As at 4 February 2020, 20,471 confirmed cases of novel coronavirus (2019-nCoV) have been reported from China with 425 deaths confirmed so far [1]. There were cases in at least 23 other countries, identified because of symptoms and recent travel history to Hubei province, China. This strongly suggests that the reported cases constitute only a small fraction of the actual number of infected individuals in China [2]. While the most affected region, Hubei province, has ceased air travel and closed major public transport routes [3] the number of exported cases are still expected to increase [4].

Despite limited evidence for its effectiveness, airport screening has been previously implemented during the 2003 SARS epidemic and 2009 influenza $A\left(\mathrm{H}_{1} \mathrm{~N}_{1}\right)$ pandemic to limit the probability of infected cases entering other countries or regions [5-7]. Here we use the available evidence on the incubation time, hospitalisation time and proportion of asymptomatic infections of 2019-nCoV to evaluate the effectiveness of exit and entry screening for detecting travellers entering Europe with 2019-nCoV infection. We also present an online tool so that results can be updated as new information becomes available.

\section{Simulation of travellers at each stage of} infection with 2019-nCoV

We simulated 100 2019-nCoV infected travellers planning to board a flight who would pose a risk for seeding transmission in a new region. The duration of travel was considered as the flight time plus a small amount of additional travel time (ca 1 hour) for airport procedures. We assumed that infected individuals will develop symptoms, including fever, at the end of their incubation period (mean 5.2 days (Table)) [8] and progress to more severe symptoms after a few days, resulting in hospitalisation and isolation. We also took into account that individuals may have asymptomatic (subclinical) infection that would not be detected by thermal scanning or cause them to seek medical care, although these individuals may be infectious, and that infected travellers may exhibit severe symptoms during their travel and be hospitalised upon arrival without undergoing entry screening. We then estimated the proportion of infected travellers who would be detected by exit and entry screening, develop severe symptoms during travel, or go undetected, under varying assumptions of: (i) the duration of travel; (ii) the sensitivity of exit and entry screening; (iii) the proportion of asymptomatic infections; (iv) the incubation period and ( $v$ ) the time from symptom onset to hospitalisation (Table).

We assume that the time of starting travel is randomly and uniformly distributed between the time of infection and twice the expected time to severe disease, ensuring that simulated travellers are travelling during their incubation period. However, we only consider those travellers who depart before their symptoms progress to being so severe that they would require hospital care [8]. We simulate travellers with individual incubation period, time from onset to severe disease, flight start times and detection success at exit and entry screening according to the screening sensitivities (Figure 1). An individual will be detected at exit screening if their 


\section{TABLE}

Parameter values and assumptions for the baseline scenario estimating effectiveness of exit and entry screening at airports for detecting passengers infected with novel coronavirus (2019-nCoV)

\begin{tabular}{|c|c|c|}
\hline Parameter & Value (baseline scenario) & Source \\
\hline Duration of travel & 12 hours & Beijing - London [18] \\
\hline Sensitivity of exit screening & $86 \%$ & Sensitivity of infrared thermal image scanners [19] \\
\hline $\begin{array}{l}\text { Proportion of asymptomatic infections undetectable } \\
\text { by typical screening procedures }\end{array}$ & $17 \%$ & $\begin{array}{l}1 \text { of } 6 \text { reported asymptomatic in a 2019-nCoV family } \\
\text { cluster [11] }\end{array}$ \\
\hline Incubation period & $\begin{array}{l}\text { Mean } 5.2 \text { days, variance } \\
4.1 \text { days }\end{array}$ & $\begin{array}{l}\text { Reported Gamma distributed mean, variance estimated } \\
\text { from uncertainty interval of mean [8] }\end{array}$ \\
\hline Time from symptom onset to hospitalisation & $\begin{array}{l}\text { Mean } 9.1 \text { days, variance } \\
14.7 \text { days }\end{array}$ & $\begin{array}{l}\text { Reported Gamma distributed mean, variance estimated } \\
\text { from uncertainty interval of mean [8] }\end{array}$ \\
\hline
\end{tabular}

\section{FIGURE 1}

Simulated infection histories of travellers infected with novel coronavirus (2019-nCoV)

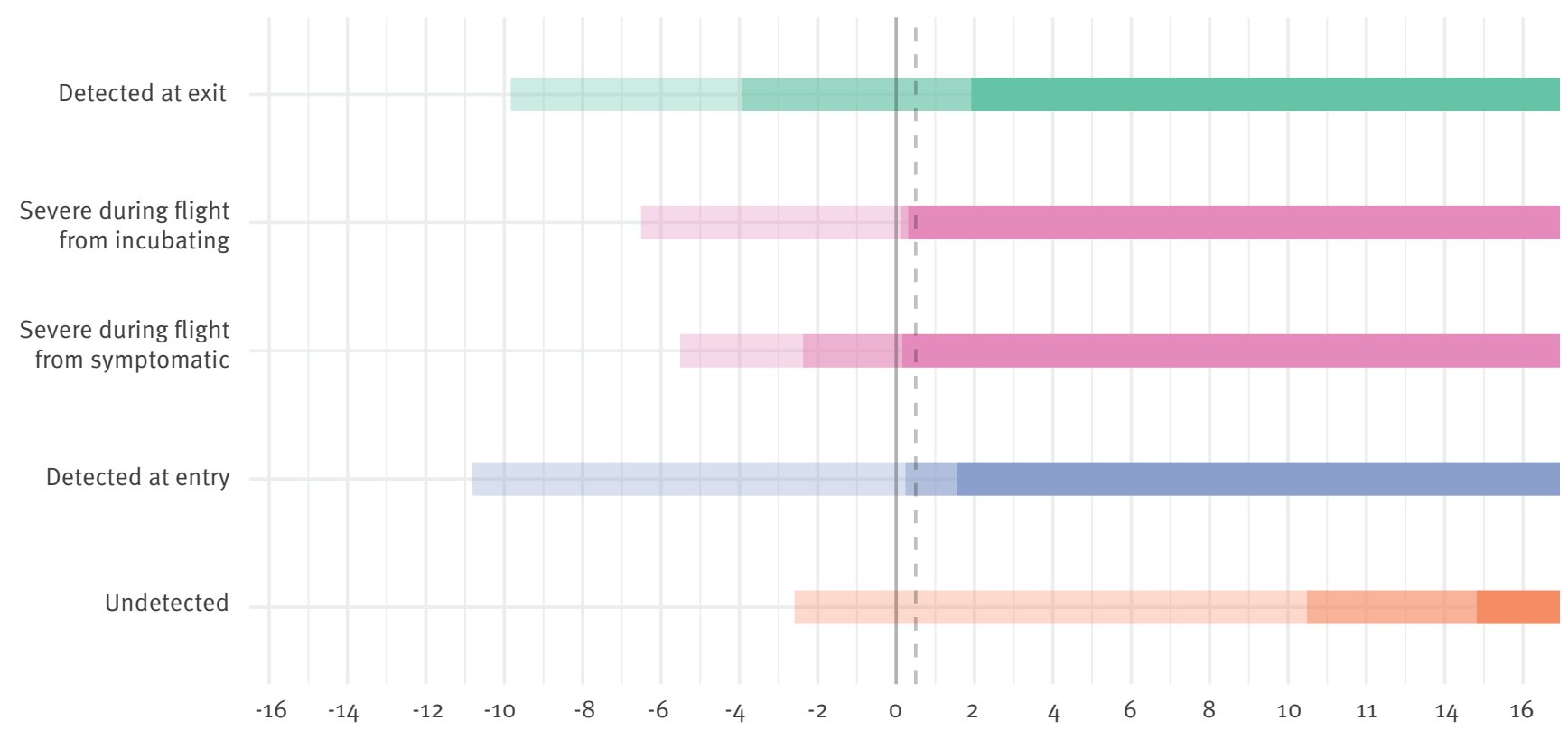

Days since flight departure

Period Incubation Symptomatic Severe

The incubation period begins on infection and travellers then progress to being symptomatic and having severe symptoms. Travellers may fly at any point within the incubation or symptomatic phases; any would-be travellers who show (severe) symptoms and are hospitalised before exit. Vertical lines represent the exit screening at start of travel (solid) and entry screening at end of travel (dashed) 12 hours later. 


\section{FIGURE 2}

Screenshot of Shiny app ${ }^{\text {a }}$ displaying the number of travellers infected with novel coronavirus (2019-nCoV) detected at airport exit and entry screening with baseline assumptions ${ }^{\mathrm{b}}$, $95 \%$ bootstrap confidence intervals, time distributions for incubation period and time to severe disease ${ }^{\star}$

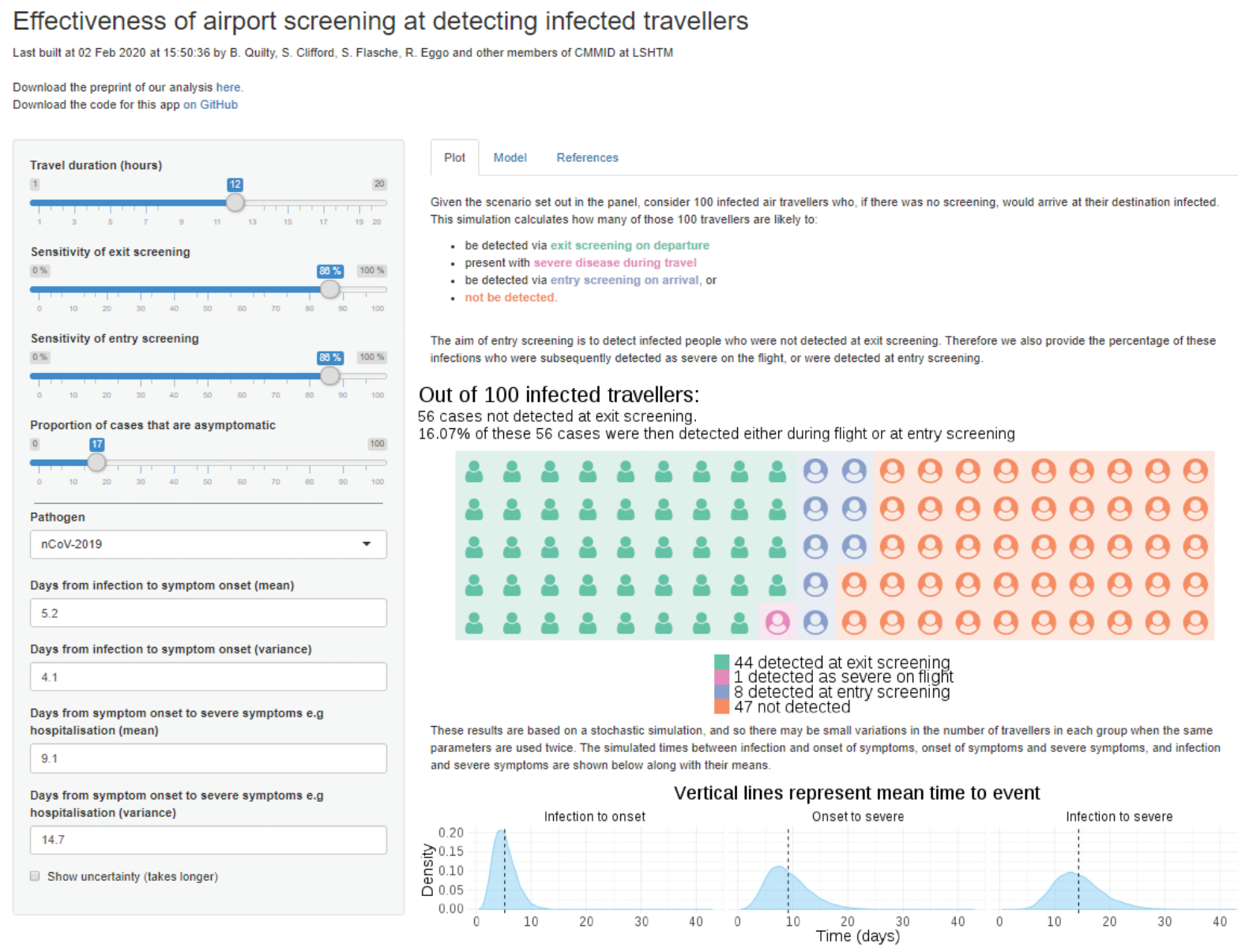

${ }^{a}$ Source [9].

${ }^{\mathrm{b}}$ Baseline assumptions according to the Table.

Results are from stochastic simulation, and so there may be small variations in the number of travellers in each group when the same parameters are used twice. Sliders are provided to modify the duration of travel, the sensitivity of both exit and entry screening, the proportion symptomatic, and the natural history parameters for the infection.

infection is symptomatic i.e. has detectable fever, their departure time exceeds their incubation period, and their stochastic exit screening success indicates detection. An individual will be detected at entry screening if their infection is symptomatic, their incubation period ends after their departure but before their arrival, they have not been detected at exit screening, and their entry screening result is positive despite imperfect sensitivity. Entry screening detections are further divided into detection due to severe symptoms and detection of mild symptoms via equipment such as thermal scanners. We used 10,000 bootstrap samples to calculate 95\% confidence intervals $(\mathrm{Cl})$.

The model code is available via GitHub [9] and the results can be further explored in a Shiny app [10] at https://cmmid-Ishtm.shinyapps.io/traveller_screening/ (Figure 2).

\section{Effect of screening on detection}

For the baseline scenario we estimated that 44 (95\% $\mathrm{Cl}: 33-56)$ of 100 infected travellers would be detected by exit screening, no case $(95 \% \mathrm{Cl}: 0-3)$ would develop severe symptoms during travel, nine $(95 \% \mathrm{Cl}: 2-16)$ additional cases would be detected by entry screening, and the remaining $46(95 \% \mathrm{Cl}: 36-58)$ would not be detected.

The effectiveness of entry screening is largely dependent on the effectiveness of the exit screening in place. Under baseline assumptions, entry screening could detect 53 (95\% Cl: 35-72) instead of nine infected travellers if no exit screening was in place. However, the probability of developing symptoms during the flight increases with flight time and hence exit screening is more effective for longer flights (Figure 3). 


\section{FIGURE 3}

Probability of detecting travellers infected with novel coronavirus (2019-nCoV) at airport entry screening by travel duration and sensitivity of exit screening

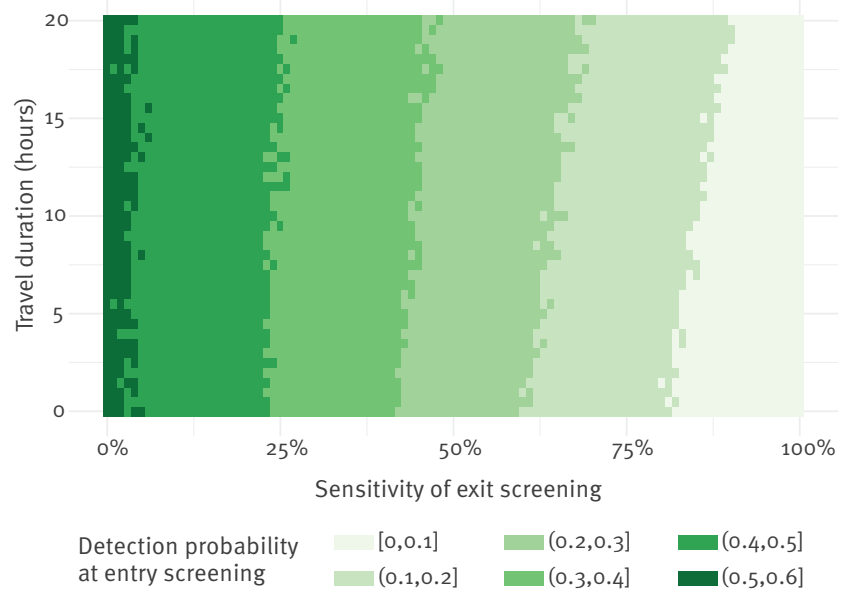

Each cell is a mean of 10,000 model simulations. Other parameters (incubation period, symptom onset to hospitalisation period, and proportion of asymptomatic infections) were fixed at baseline assumptions (Table). Intervals are probabilities of detection, binned at increments of $10 \%(0-10 \%, 10-20 \%$, etc.).

Syndromic screening designed to prevent infected and potentially infectious cases entering a country undetected is highly vulnerable to the proportion of asymptomatic infections and long incubation periods. If our baseline scenario is modified to have o\% asymptomatic 2019-nCoV infections and $100 \%$ sensitivity of entry screening, the incubation period will need to be around 10 -fold shorter than the period from symptom onset to severe disease (e.g. hospitalisation) in order to detect more than $90 \%$ of infected travellers that would not otherwise report illness at either exit or entry screening.

\section{Discussion and conclusions}

As a response to the ongoing outbreak of the 2019$\mathrm{nCoV}$ originating in Wuhan, exit screening has been implemented for international flights leaving China's major airports. Thermal scanning, which can identify passengers with fever (high external body temperature), allows for passengers exhibiting symptoms of 2019-nCoV infection to be tested before they board a plane. Similarly, entry screening for flights originating in the most affected regions may be under consideration at airports in regions in and outside China. We estimate that the key goal of syndromic screening at airports - to prevent infected travellers from entering countries or regions with little or no ongoing transmission - is only achievable if the rate of asymptomatic infections that are transmissible is negligible, screening sensitivity is almost perfect, and the incubation period is short. Based on early data from Li et al. [8], 2019-nCoV appears to have a shorter incubation period than severe acute respiratory syndrome (SARS), and a higher rate of asymptomatic infections [11]. Under generally conservative assumptions on sensitivity, we find that 46 of 100 infected travellers will enter undetected.

Entry screening is an intuitive barrier for the prevention of infected people entering a country or region. However, evidence on its effectiveness remains limited and given its lack of specificity, it generates a high overhead of screened travellers uninfected with the targeted pathogen [5]. For example, when entry screening was implemented in Australia in response to the 2003 SARS outbreak, 1.84 million people were screened, 794 were quarantined, and no cases were confirmed [12]. While some cases of 2019-nCoV infection have been identified through airport screening in the current outbreak, our estimates indicate that likely more infected travellers have not been detected by screening.

It is important to note that our estimates are based on a number of key assumptions that cannot yet be informed directly by evidence from the ongoing 2019nCoV outbreak. The current outbreak has spread rapidly and early evidence suggests that the average disease severity is lower than that of SARS. This may also suggest a substantial proportion of asymptomatic cases. A recent analysis of a family transmission cluster is based on a small sample size but one in six infections was asymptomatic [11]; this is a major impediment for the effectiveness of syndromic screening. However, if asymptomatic cases were not infectious they would not pose a risk for seeding infection chains on arrival. To allow easy adaptation of our results as new insight becomes available in the coming weeks, we developed a free interactive online tool, available at https:// cmmid-Ishtm.shinyapps.io/traveller_screening/.

While the most up-to-date data on the incubation period or the time until recovery from 2019-nCoV infection have been used in this analysis, these figures are likely to change over time as more data become available. Unless the incubation period is only a small fraction of the duration of infection in relation to that of symptomatic disease, and fever in particular, syndromic screening is likely to detect an insufficient fraction of infected cases to prevent local infections. In addition, the sensitivity of airport screening for the detection of 2019-nCoV has not been evaluated. However, we chose conservative estimates and show that with reduced sensitivity, the effectiveness of syndromic screening would further decline.

In many international airports, information is provided to travellers from affected regions recommending action if they develop symptoms on or after arrival [1316]. Some countries, for example Japan, also require incoming passengers to complete forms detailing their past and future travel in order to aid tracing [17]. Due to the duration of the incubation period of $2019-\mathrm{nCoV}$ infection, we find that exit or entry screening at airports for initial symptoms, via thermal scanners or similar, is unlikely to prevent passage of infected travellers into 
new countries or regions where they may seed local transmission.

\section{${ }^{\star}$ Erratum}

Figure 2 was replaced on 7 February 2020.

Members of the Centre for the Mathematical Modelling of Infectious Diseases (CMMID) $\mathrm{nCoV}$ working group

Yang Liu, Charlie Diamond, W John Edmunds, Sebastian Funk, Amy Gimma, James D Munday, Hamish Gibbs, Nikos I Bosse, Sam Abbott, Timothy W Russell, Petra Klepac, Mark Jit, Joel Hellewell.

\section{Acknowledgements}

SF and SC are supported by a Sir Henry Dale Fellowship jointly funded by the Wellcome Trust and the Royal Society (Grant number $208812 / Z / 17 / Z$ ).

RME acknowledges an HDR UK Innovation Fellowship (Grant number MR/So03975/1).

BJQ was funded by the National Institute for Health Research (NIHR) $(16 / 137 / 109)$ using UK aid from the UK Government to support global health research. The views expressed in this publication are those of the author(s) and not necessarily those of the NHS, the NIHR or the UK Department of Health and Social Care.

\section{CMMID nCoV working group funding statements:}

Yang Liu (Gates (INV-003174), NIHR (16/137/109)), Charlie Diamond (NIHR (16/137/109)), Sebastian Funk (Wellcome Trust (210758/Z/18/Z)), Amy Gimma (Global Challenges Research Fund (GCRF) for the project "RECAP" managed through RCUK and ESRC (ES/Po10873/1)), James D Munday (Wellcome Trust (210758/Z/18/Z)), Hamish Gibbs (NIHR (ITCRZ 03010)), Sam Abbott (Wellcome Trust (210758/Z/18/Z)), Timothy W Russell (Wellcome Trust (206250/Z/17/Z)), Petra Klepac (Gates (INV003174)), Mark Jit (Gates (INV-003174), NIHR (16/137/109)), Joel Hellewell (Wellcome Trust (210758/Z/18/Z)).

\section{Conflict of interest}

None declared.

\section{Authors' contributions}

Conceptualisation: BJQ, SF, SC, RME; model formulation: SC, BJQ, SF; analysis: BJQ, SC; writing: RME, SF, SC, BJQ; app testing: RME and the Centre for the Mathematical Modelling of Infectious Diseases (CMMID) nCoV working group. The members of the CMMID nCoV working group contributed equally in processing, data cleaning, interpreting findings, testing the interactive tool, reviewing the manuscript and approving the work for publication. The order was assigned randomly.

\section{References}

1. World Health Organization (WHO). Novel Coronavirus (2019nCoV) Situation Report-15. Geneva: WHO; 4 Jan 2020. Available from: https://www.who.int/docs/default-source/ coronaviruse/situation-reports/20200204-sitrep-15-ncov. pdf?sfvrsn=88fe8ad6_2
2. Imperial College London. News / Wuhan Coronavirus. MRC Centre for Global Infectious Disease Analysis: News / Wuhan Coronavirus. London: Imperial College London. [Accessed 27 Jan 2020]. Available from: http://www.imperial.ac.uk/ medicine/departments/school-public-health/infectiousdisease-epidemiology/mrc-global-infectious-disease-analysis/ news--wuhan-coronavirus/

3. Reuters. Wuhan lockdown 'unprededented', shows commitment to contain virus: WHO representative in China. [Accessed 4 Feb 2020]. Available from: https://www.reuters.com/article/ us-china-health-who-idUSKBN1ZM1G9

4. Kucharski AJ, Russell TW, Diamond C. CMMID nCoV working group, Funk S, Eggo RM. Early dynamics of transmission and control of 2019-nCoV: a mathematical modelling study (preprint). Medrxiv. 2020. http://dx.doi.org/ https://doi.org/10.110 1/2020.01.31.20019901

5. Gostic KM, Kucharski AJ, Lloyd-Smith JO. Effectiveness of traveller screening for emerging pathogens is shaped by epidemiology and natural history of infection. eLife. 2015;4:e05564. https://doi.org/10.7554/eLife.05564 PMID: 25695520

6. Mabey D, Flasche S, Edmunds WJ. Airport screening for Ebola. BMJ. 2014;349(oct14 17):g6202. PMID: 25316030

7. Pitman RJ, Cooper BS, Trotter CL, Gay NJ, Edmunds WJ. Entry screening for severe acute respiratory syndrome (SARS) o influenza: policy evaluation. BMJ. 2005;331(7527):1242-3. https://doi.org/10.1136/bmj.38573.696100.3A PMID: 16176938

8. Li Q, Guan X, Wu P, Wang X, Zhou L, Tong Y, et al. Early Transmission Dynamics in Wuhan, China, of Novel CoronavirusInfected Pneumonia. N Engl J Med. 2020;NEJMoa2001316. PMID: 31995857

9. GitHub. Traveller Screening GitHub Repository. San Francisco: GitHub. [Accessed 5 Feb 2020]. Available from: https://github. com/bquilty25/airport_screening

10. Chang W, Cheng J, Allaire JJ, Xie Y, McPherson J. shiny: Web Application Framework for R. R package version 1.4.0. 2019. [Accessed 27 Jan 2020]. Available from: https://CRAN.Rproject.org/package $=$ shiny

11. Chan JF-W, Yuan S, Kok K-H, To KK-W, Chu H, Yang J, et al. A familial cluster of pneumonia associated with the 2019 novel coronavirus indicating person-to-person transmission: a study of a family cluster. Lancet. 2020;0(0):S0140-6736(20)30154-9. PMID: 31986261

12. Samaan G, Patel M, Spencer J, Roberts L. Border screening for SARS in Australia: what has been learnt? Med J Aust. 2004;180(5):220-3. https://doi.org/10.5694/j.1326-5377.2004. tbo5889.x PMID: 14984341

13. European Centre for Disease Prevention and Control (ECDC). Advice for travellers: outbreak of a novel coronavirus 2019-nCoV. Stockholm: ECDC. 30 Jan 2020. Available from: https://www.ecdc.europa.eu/en/publications-data/ advice-travellers-outbreak-novel-coronavirus-2019-ncov

14. San Francisco International Airport. Passengers arriving from Wuhan, China are being screened for the novel coronavirus. San Francisco: San Francisco International Airport. [Accessed 27 Jan 2020]. Available from: https://www.flysfo.com/ passengers-arriving-wuhan-china-are-being-screened-novelcoronavirus

15. Department of Health. Coronavirus: latest information and advice. London: GOV.UK. [Accessed 27 Jan 2020]. Available from: https://www.gov.uk/guidance/wuhan-novel-coronavirusinformation-for-the-public\#advice-for-travellers-from-wuhan

16. DiGiovanni C, Conley J, Chiu D, Zaborski J. Factors influencing compliance with quarantine in Toronto during the 2003 SARS outbreak. Biosecur Bioterror. 2004;2(4):265-72. https://doi. org/10.1089/bsp.2004.2.265 PMID: 15650436

17. The Japan Times. Countries around world gear up response to new coronavirus. 2020. [Accessed 5 Feb 2020]. Available from: https://www.japantimes.co.jp/ news/2020/01/22/asia-pacific/science-health-asia-pacific/ countries-around-world-gear-response-new-coronavirus/

18. British Airways. Timetables. British Airways. [Accessed 27 Jan 2020]. Available from: https://www.britishairways.com/travel/ schedules/public/en_gb

19. Priest PC, Duncan AR, Jennings LC, Baker MG. Thermal image scanning for influenza border screening: results of an airport screening study. PLoS One. 2011;6(1):e14490. https://doi. org/10.1371/journal.pone.0014490 PMID: 21245928 
License, supplementary material and copyright

This is an open-access article distributed under the terms of the Creative Commons Attribution (CC BY 4.0) Licence. You may share and adapt the material, but must give appropriate credit to the source, provide a link to the licence and indicate if changes were made.

Any supplementary material referenced in the article can be found in the online version.

This article is copyright of the authors or their affiliated institutions, 2020. 